\title{
Finding meaning in medical school
}

Previously published at www.cmaj.ca

The Mindful Medical Student: A

Psychiatrist's Guide to Staying Who You Are While Becoming Who You Want to Be Jeremy Spiegel MD

Dartmouth College Press; 2009.

$\mathrm{S}$ leep deprivation, competitive classmates, patronizing attending physicians, challenging patients, ethical dilemmas, stressful exams - these are all common aspects of the medical school experience. In The Mindful Medical Student, psychiatrist Jeremy Spiegel does not attempt to make medical school any less demanding. Rather, he focuses on assessing how the process can be more personally meaningful, so that it becomes something to appreciate rather than something to be endured.

Of the book's three sections - "Discovering who you are," "Reinventing yourself" and "Blazing a path to your deeper self"- Spiegel is most successful in the first. The development of greater self-awareness, Spiegel argues, is fundamental to a healthy journey through medical training. The themes of Spiegel's book centre on the notion of a multifaceted identity. He describes a "true self" and a "false" (or performing) self and how, through conscious effort, a student can learn to nurture the former and deconstruct the latter. Balancing the theoretical discussions are vivid real-life anecdotes from medical students.

Spiegel takes a Freudian psychoanalysis-lite approach to common experiences, framing his discussion in terms of transference, defences and the unconscious. One example is the humorously entitled chapter "Dream Interpretation for First-Time Scalpel

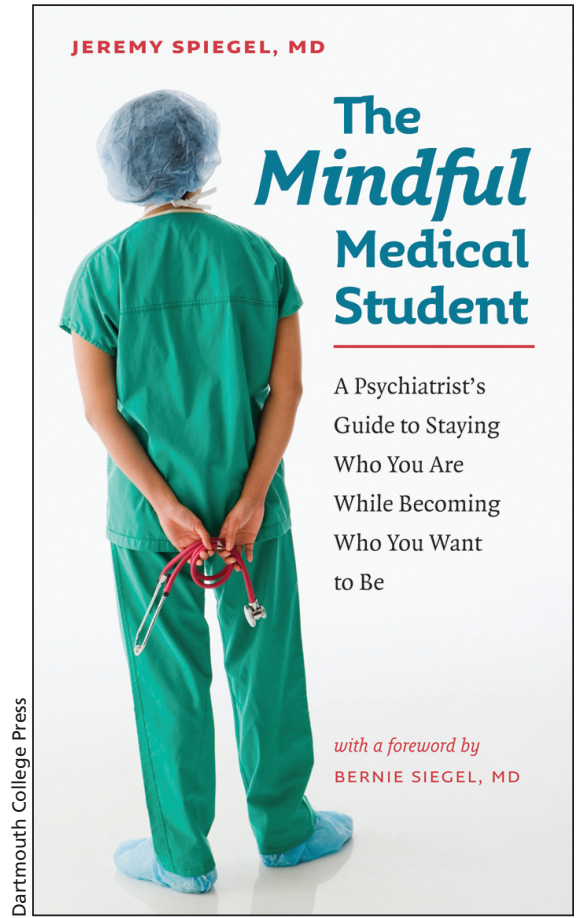

Wielders." Another chapter about archetypes and synchronicities has a more Jungian slant.

Other chapters deal with more concrete issues, such as managing your perfectionist or obsessive-compulsive tendencies and learning how to form supportive bonds with classmates.

Spiegel acknowledges that certain traits that may have helped students get into medical school can become maladaptive under stressful circumstances, and offers several pragmatic tips on how to address these issues. These are typically simple mental exercises that one can practise anywhere at anytime, be it on the ward or in a call room. However, some suggestions will be a stretch for the average students. For example, Spiegel suggests creating masks and then roleplaying the different personas one encounters on the wards, such as "the abysmal anti-mentor," "the somatizing drama queen" and "the boundarydeficient love seeker."

What sets The Mindful Medical Student apart from similarly themed books is its emphasis on achieving clarity, reflective capacity and wholeness through the creation of a uniquely personal narrative. Spiegel's techniques facilitate the processing of experiences so students take an active role in moulding their own transformation and protecting themselves against emotional shutdown and cynicism.

Spiegel draws on literary, philosophical and spiritual sources to combat what he refers to as "right brain atrophy" and "relationship anemia." The goal is to move away from compartmentalized identities and rigid defenses, and to develop a more integrated and consistent personality, one that is capable of treating self, patients and colleagues with equal compassion.

The Mindful Medical Student is an enjoyable read with an entertaining conversational style. Despite its title, the book shares valuable insights that may be useful for all health care providers in training. It is essentially a manual on how to maintain sanity in an often gruelling medical environment and culture, makes a compelling argument that self-care is not a luxury. It gives students permission to step back from their textbooks, to distinguish the forest from the trees and to appreciate their special place in that forest.

\section{Denise Sum BASc \\ Class of 2010 \\ Faculty of Medicine \\ McMaster University \\ Hamilton, Ont.}

